\title{
CAUSAS DE ÓBITO EM PACIENTES PÓS-TRANSPLANTE HEPÁTICO IMEDIATO E TARDIO DE UM SERVIÇO DE REFERÊNCIA EM SANTA CATARINA
}

\author{
Causes of death in immediate and late liver post-transplantation patients \\ in a reference service in Santa Catarina
}

\begin{abstract}
Neide da Silva Knihs, Rosi Meri da Silva Longo, Emanuela Ueno, Maria Aparecida Kratz Leite, Mauro Rafael da Igreja, Marcelo Augusto Scheidemantel Nogara
\end{abstract}

\begin{abstract}
RESUMO
Objetivo: identificar as causas dos óbitos imediatos e tardios em pacientes submetidos a transplante hepático no período de 2002 a 2007 em um hospital de referência no estado de Santa Catarina. Método: Trata-se de uma pesquisa quantitativa, retrospectiva de caráter descritivo, abrangendo o período de agosto de 2002 a dezembro de 2007. Fizeram parte da amostra 51 prontuários de pacientes que evoluíram a óbito nesse período. Resultados: Mostram como principal causa de óbito no pós-operatório imediato o choque séptico e o choque hemorrágico, com média de sobrevida de 23,7 dias. Em relação ao óbito tardio, a principal causa foi o choque séptico, com média de sobrevida de 244,1 dias. Com relação à idade, os óbitos ocorreram entre 40 e 60 anos, predominando o óbito no sexo masculino. Conclusões: Através do presente estudo, foi possível identificar que a maior causa de óbito no transplante hepático foi o choque séptico, tendo como principal intercorrência a pneumonia. A faixa etária mais atingida foi entre os 40 e 60 anos, com predominância do sexo masculino.
\end{abstract}

Descritores: Transplante hepático, Causa de Óbito, Transplante, Hepatopatias.

Instituição:

Departamento de Transplante da Sociedade Divina Providencia Hospital Santa Isabel Blumenau / SC

\section{Correspondência:}

Neide da Silva Knihs

Rua Victor Meirelles, 63 - Brusque / SC - CEP: 88351-700 - Brasil

Tel.: (47) 33553409

E-mail: ajknihs@gmail.com

Recebido em: 11.06 .2009

Aceito em: 03.07.2009

\section{INTRODUÇÃO}

O transplante hepático tem sido considerado um dos procedimentos mais complexos na área da medicina por interferir em várias funções do organismo. A excelência no seu resultado está relacionada à infra-estrutura hospitalar adequada e a uma equipe interdisciplinar altamente capacitada para atuar no procedimento e acompanhamento pré e pós-transplante. ${ }^{1}$

Nos últimos anos, os transplantes hepáticos têm apresentado grande progresso quanto a seus resultados. O estado de Santa Catarina, um dos centros de referência do Brasil, iniciou suas atividades de transplante hepático no ano de 2002. Desde então, vem aumentando a cada ano o número de transplantes, possibilitando assim um aprimoramento técnico e científico dos profissionais que atuam nas equipes cirúrgica e clínica. Acrescenta-se a isso o acompanhamento do paciente desde a indicação para o transplante, o período de espera, o período pós-transplante, a alta hospitalar e o atendimento em domicílio pela equipe interdisciplinar.

A equipe deve considerar a avaliação clínica no período que antecede o transplante, identificando fatores que possam ocasionar complicações no pós-operatório na tentativa de minimizar a incidência dessas intercorrências. Dentre os principais fatores a serem observados estão a desnutrição, agravamento das funções hepáticas e renais, as alterações imunológicas, o comprometimento do sistema cardiovascular, cardiorrespiratório, neurológico e psicológico. O fator importante a ser observado é a idade do paciente, uma vez que, pacientes acima de 65 anos apresentam maior risco operatório e recuperação pós-operatória mais lenta. ${ }^{1}$ 
A causa do óbito pode estar direta ou indiretamente relacionada a esses fatores mencionados. Entretanto, intercorrências e alterações hemodinâmicas importantes podem acontecer durante o intraoperatório, mesmo diante de todo esforço da equipe em tentar minimizar os riscos nesse momento.

Sabe-se que o transplante de fígado é considerado hoje uma das maiores cirurgias do corpo humano. Aliado a isso, na maioria dos casos, o paciente apresenta cirrose hepática, plaquetopenia, distúrbio de coagulação. Outro fator importante é o tempo de espera em lista, que pode agravar ainda mais suas condições clínicas, podendo assim, prolongar ainda mais o tempo cirúrgico e as complicações no intra e pós-operatório. ${ }^{2,3}$

Outro fator importante a ser considerado é o uso de medicamentos imunossupressores, os quais agem no organismo evitando a rejeição. Contudo, esses medicamentos também podem trazer uma série de alterações no organismo, tornando os pacientes susceptíveis a desenvolver nefrotoxidade, neurotoxidade, neutropenia e outras complicações que podem levar a um aumento da gravidade da evolução clínica e até mesmo a óbito. Soma-se a isso o surgimento de doenças oportunistas, configurando-se em um aumento dos casos de óbitos nos pacientes transplantados, não somente de fígado, como também em outros órgãos sólidos transplantados. ${ }^{3-5}$

Frente ao exposto, sendo o único centro credenciado no estado de Santa Catarina para realização de transplante hepático, sentiu-se necessidade de identificar as causas dos óbitos imediatas e tardias em pacientes submetidos a transplante hepático no período de agosto de 2002 a dezembro de 2007. Nesse período, foram realizados 191 transplantes, dentre os quais 60 pacientes foram a óbito.

\section{MÉTODO}

Trata-se de pesquisa quantitativa, retrospectiva de caráter descritivo, abrangendo o período de agosto de 2002 a dezembro de 2007. A amostragem foi estratificada e foram levantadas na coleta de dados as seguintes variáveis: idade, sexo, diagnóstico inicial, infecções, intercorrências, causa do óbito, tempo de sobrevida.

O presente estudo foi desenvolvido no Serviço de Transplante Hepático do Hospital Santa Isabel. Trata-se de um hospital de alta-complexidade do município de Blumenau, estado de Santa Catarina, considerado como referência nacional e única instituição credenciada para esse serviço neste estado.

A população do estudo foi composta por prontuários de pacientes que evoluíram para óbito após terem sido submetidos ao transplante de fígado no Hospital Santa Isabel, no período de agosto de 2002 a dezembro de 2007. Para a seleção da amostra, buscaram-se todos os óbitos registrados através do ambulatório de transplantes, num total de 60 óbitos. Em seguida, a busca foi realizada através do arquivo dos prontuários, totalizando 51 prontuários. Nove prontuários de “óbito” não foram localizados, por se tratar de pacientes que foram a óbito no domicilio e em outras instituições hospitalares.

Para coleta de dados nos prontuários foi estruturado um instrumento de coleta de dados contendo questões fechadas (10) e abertas (6), elaborado a partir da revisão da literatura nacional e internacional sobre os sujeitos do estudo. Das questões fechadas, duas faziam parte do perfil da população e as outras 14 abordaram informações relacionadas à patologia e às causas que contribuíram para o óbito do paciente. Foi realizado pré-teste em cinco prontuários, para ajustar o questionário aos objetivos do estudo.
Os dados foram coletados no período de janeiro a abril de 2008 pelos próprios pesquisadores, após apresentação e aprovação da pesquisa ao Comitê de Ética. A solicitação dos prontuários foi feita à instituição, os quais foram separados por ano. Em seguida, foi realizada a coleta dos dados. As dificuldades encontradas durante a coleta de dados estavam relacionadas a letras ilegíveis dos profissionais durante as evoluções, uma vez que aqueles prontuários não eram digitalizados.

A análise dos dados foi organizada primeiramente em banco de dados (Microsoft Excel); na seqüência, foram confeccionados primeiramente gráficos individuais, e, posteriormente, foi feito cruzamento dos dados para novos gráficos, para que assim fosse possível analisar os dados contidos nesses gráficos.

Todos os dados coletados estavam sob responsabilidade dos pesquisadores, respeitando os regulamentos da Resolução n 196/96.

\section{RESULTADOS}

Dos 174 pacientes que foram submetidos a transplante hepático entre agosto de 2002 e dezembro de 2007, 60 pacientes foram a óbito. Desses, 43 foram a óbito imediato (ou seja, entre um e 120 dias após o transplante), oito evoluíram a óbito tardio (ou seja, após 120 dias de transplante) e nove foram a óbito em seu domicílio ou em outra instituição de saúde.

O choque séptico foi a causa de maior predominância, sendo observado em $17(39,6 \%)$ pacientes no óbito imediato e em seis (75\%) no óbito tardio. No óbito imediato, a segunda causa principal foi o choque hemorrágico em nove casos $(20,9 \%)$, seguida de falência do enxerto ocorrida em seis casos $(13,9 \%)$. Cinco pacientes $(11,7 \%)$ foram a óbito por rejeição e seis $(13,9 \%)$ foram agrupados como outras causas, incluindo-se insuficiência cardíaca, Síndrome de Angústia Respiratória do Adulto (SARA) e distúrbios de coagulação. No óbito tardio, a segunda maior causa foi falência do enxerto. A principal intercorrência que levou ao desenvolvimento de choque séptico foi insuficiência respiratória, tendo como conseqüência a pneumonia. Outras intercorrências que surgiram foram infecção do trato urinário e infecção da ferida cirúrgica. Dos pacientes que evoluíram para choque séptico no óbito imediato, oito $(18,6 \%)$ apresentaram dispnéia, tosse e pneumonia, havendo a necessidade de reintubação. Cinco pacientes (11,6\%) apresentaram edema agudo, sobrecarga cardíaca e insuficiência respiratória, seguidos de choque séptico.

Em relação ao choque hipovolêmico, as intercorrências que mais estiveram presentes foram: sangramento no pós-operatório nas primeiras 24 horas, seguido de retransplante em dois casos e hemorragia. Dos pacientes que evoluíram para choque hemorrágico, cinco (11,6\%) tiveram intraoperatório complicado, apresentando hipotensão severa, sangramento, óligúria, choque hipovolêmico e óbito.

Em relação à falência do enxerto e rejeição, estavam presentes como principais complicações: insuficiência renal aguda, insuficiência respiratória, trombose da artéria hepática e veia cava, retransplante.

No óbito tardio, no que se refere ao choque séptico, a pneumonia esteve presente em cinco (62,5\%) pacientes. Estes pacientes chegaram ao serviço de emergência apresentando tosse, dispnéia, seguida de insuficiência respiratória aguda. Um dos pacientes que evoluiu para choque séptico chegou ao serviço de emergência apresentando abscesso abdominal, sendo realizada drenagem da cavidade abdominal, com saída de grande quantidade de secreção e presença 
de ascite (peritonite, seguido de choque séptico). Em relação à falência do enxerto, a insuficiência renal aguda, seguida de insuficiência respiratória e retransplante, foram as principais complicações.

O tempo de sobrevida no óbito tardio variou de 120 a 520 dias, média de 244,1 dias; faixa etária de predomínio entre 50 a 60 anos (62,5\%). No óbito imediato, o tempo de sobrevida foi de um a 120 dias, com média de 23,7 dias; faixa etária de predomino de 41 a 50 anos (32,5\%) e 51 a 60 anos (37,2\%). Em relação ao gênero, houve maior predomínio do sexo masculino tanto no óbito imediato 24 (55,8\%), quanto no óbito tardio 6 (75\%). Dos 43 pacientes que evoluíram para óbito imediato, 17 (39,5\%) tinham como patologia de base o vírus da hepatite tipo C e 11 (25,5\%) cirrose por álcool.

\section{DISCUSSÃO}

O transplante hepático basicamente tem por objetivo prolongar a vida do paciente, proporcionando qualidade de vida satisfatória e recuperação da capacidade de trabalho. O aprimoramento das técnicas aliado ao uso de novos fármacos tem favorecido esse resultado.

O transplante hepático é considerado uma das maiores cirurgias do corpo humano, agregando assim um tempo cirúrgico significativo para que ocorra o procedimento. O tempo de isquemia a que esse órgão a ser transplantado é submetido, somado a necessidade de uso de imunossupressores são fatores fundamentais, que tornam o transplante de fígado tão suscetível à intercorrências, complicações e óbito dos pacientes. ${ }^{4,6,7}$

A sobrevida do paciente submetido a transplante hepático vem aumentando progressivamente nos últimos anos, alcançando média de $60-70 \%$ em cinco anos. A escolha do paciente a ser transplantado, a experiência da equipe cirúrgica e a participação da equipe interdisciplinar na minimização dos riscos têm contribuído significativamente para esse resultado.,

As intercorrências e complicações relacionadas às causas de óbito diferem conforme o tempo de sobrevida. A maioria dos óbitos ocorre nos três primeiros meses após o transplante, e são geralmente devidos a complicações técnicas da operação e ao não funcionamento primário do enxerto. ${ }^{7,9-11}$

Os resultados deste estudo mostram que o óbito dos pacientes ocorre principalmente nos primeiros trinta dias após o transplante. As intercorrências relacionadas ao óbito são variadas, entretanto, nota-se o predomínio da insuficiência respiratória, seguida de pneumonia, tanto no óbito imediato quanto no tardio. As principais causas de intercorrências por infecção hospitalar têm sido as infecções do trato respiratório. Esse risco tende a ser potencializado devido a necessidade da ventilação mecânica juntamente com o uso de imunossupressores. Esses pacientes apresentam maior risco de desenvolver infecções respiratórias quando comparados a pacientes que não são submetidos à ventilação mecânica associada a imunossupressão. ${ }^{4,12}$
No que diz respeito à causa do óbito, a literatura mostra que as infecções em transplantes são freqüentes, e estão relacionadas a diversas causas. Dentre elas destacam-se as condições clinicas do paciente no momento em que é realizado o transplante, o tempo cirúrgico, as intercorrências durante o período intra-operatório, o tempo de isquemia do órgão doador, bem como o período de internação, são fatores que podem predispor ao desenvolvimento de infecções. . $^{2,4,5,11,13}$

No presente estudo observou-se que a maior causa de óbito no pós-operatório foi o choque séptico, não obstante a profilaxia e as condutas tomadas para evitar infecções. A literatura reforça o alto índice de infecção relacionada aos transplantes de órgãos. Outro fator importante, especialmente no transplante de fígado, são as condições clínicas dos pacientes no período de espera para o transplante, juntamente com o tempo cirúrgico prolongado. ${ }^{2,4}$ Infecção permanece como a principal complicação após transplante hepático, sendo que a maioria dos casos acontece após o primeiro mês do transplante. Vale ressaltar que a morte pode acontecer em qualquer período após a cirurgia.

As condições clínicas e a idade do paciente devem ser avaliadas pela equipe interdisciplinar durante todo período de espera para a cirurgia, a fim de minimizar os riscos associados ao transplante. Em nossa realidade, houve predomínio de óbito em homens com idade entre 40 e 60 anos. A literatura consultada refere que após os 45 anos diversas complicações podem estar associadas às condições cardíacas, pulmonares e circulatórias, potencializando os riscos durante o período intra e pós operatórios, incluindo-se recuperação mais lenta ou até mesmo desenvolvimento de outras doenças. 7,10

A literatura científica tem mostrado que receptores de transplante apresentam maiores complicações perioperatórias, principalmente quando apresentam quadros clínicos prévios de ascite, encefalopatia hepática, alteração do tempo de protrombina e hiperbilirrubinemia, além de insuficiência renal. Desde 2006, o Ministério da Saúde redefiniu os critérios de alocação de órgãos para fígado por gravidade da patologia associada, potencializando o risco de complicações no período pós-operatório.,11,14 A realidade do presente estudo vem ao encontro dos dados da literatura nacional e internacional, uma vez que houve predominância de casos de óbitos em pacientes com cirrose hepática por vírus da hepatite $\mathrm{C}$ e álcool.

\section{CONCLUSÕES}

Os dados deste estudo vão ao encontro dos dados da literatura nacional e internacional no que se refere ao tempo de sobrevida e as complicações relacionadas às causas de óbito. Todavia, diferem da causa de óbito que tinha como principal resultado choque séptico tanto no pós-operatório imediato quanto no tardio. Junto a isso, foi possível identificar a prevalência de óbitos no sexo masculino, bem como maior predominância de complicações na faixa etária de 50 a 60 anos. Também foi observado que a principal patologia de base da causa dos óbitos foi cirrose por vírus da hepatite C.

\section{ABSTRACT}

Purpose: To identify causes of late and immediate death in patients submitted to liver transplantation in the period from 2002 to 2007 in a reference hospital in the state of Santa Catarina. Method: This is a retrospective, quantitative research of investigative features from August, 2002 to December, 2007. Fifty-one medical records of patients were assessed in samples evolved to death along that period. Result: Septic 
and hemorrhagic shock are shown as the major immediate cause to the post-operatory death, with average life expectation of 23.7 days. The major cause to the late death was septic shock, with average life expectation of 244.1 days. Related to the TRE age, deaths occurred between 40 to 60 years old with predominance of death among the masculine gender. Conclusions: It was possible to identify through the present study that the major cause of death in liver transplantation was septic shock, having as major intercurrence pneumonia. The more affected age was between 40 and 60 years old, with predominance of masculine gender.

Keywords: Liver Transplantation, Cause of Death, Transplantation, Liver Diseases.

\section{REFERÊNCIAS}

1. Magalhães RA, Sanches, MD, Pereira WA. Doador. In: Manual de transplantes de órgãos e tecidos. Rio de Janeiro: MEDSI Editora, 2004. p. 202-29.

2. Coelho JC, et al. Infecções hospitalares bacterianas em unidade de transplante hepático. Jornal Brasileiro de Transplantes. 2004;7:198-202.

3. Coelho JC, et al. Incidência de Infecções em unidade de transplante hepático. Registro Brasileiro de Transplante. Associação Brasileira de Transplante de Órgãos. São Paulo. 2002;3:3-20.

4. Freire MP. Infecções hospitalares em pacientes submetidos a transplante de fígado: fatores de risco relacionados ao doador. (Dissertação). São Paulo. Faculdade de Medicina Universidade de São Paulo; 2006

5. Ferraz M.LG, Schiavon J.L.N, Silva AEB. Guia de hepatologia: guia de medicina ambulatorial e hospitalar. Barueira, São Paulo: Manole, 2007.

6. Coelho JC, et al. Causa de óbito tardio em transplantados de fígado. Revista da Associação Médica Brasileira. 2003;49:80-8.
7. Oliveira DMS, Drachler ML, Oliveira LS. Fatores de risco para a falência do transplante ortotópico de fígado no Rio Grande do Sul, Brasil. Cad. de Saúde Pública. 2007;23:2-9.

8. Silva AA, et al. Transplante de Fígado. In: Dani R. Gastroentereologia Essencial. Rio de Janeiro: Guanabara Koogan;2006,795-808.

9. Silva OC, et. al. Transplante de fígado: indicação e sobrevida. Revista Acta Cirúrgica Brasileira. 2002;17(suplemento 3),83-91.

10. Kieling CO. Fatores de risco para óbito precoce em crianças e em adolescentes submetidos transplante hepático eletivo no HCPA (Dissertação de Mestrado). Porto Alegre: Universidade Federal do Rio Grande do Sul; 2002.

11. Pereira WA, et al. Transplante de Fígado. In: Manual de transplantes de órgãos e tecidos. Rio de Janeiro: MEDSI Editora; 2004; 299-357.

12. Silva E. Sepse: Manual. São Paulo: editora Atheneu; 2006

13. Mendes, KDS, Galvão, CM. Transplante de Fígado: evidências para o cuidado de enfermagem. Re. Latino-Am. Enfermagem. Ribeirão Preto. 2008; 16: 915-922.

14. Coelho JCU. et al. Complicações Vasculares Pós-transplante Hepático. Revista do Colégio Brasileiro de Cirurgiões. 2000; 27:378-82. 